Artigo Original

\title{
Identificação de papéis ocupacionais em atletas do esporte adaptado
}

\section{Identification of occupational roles in adapted sport athletes}

\author{
Ana Cláudia da Silva de Sousa ${ }^{a}$ (D), Érika Vanessa Freire Frasson ${ }^{a}$ (D), Solena Ziemer Kusmaa,b (D), \\ Maria de Fátima Fernandes Vara, (D), Aline Ferrari Fabri ${ }^{\mathrm{a}}$ (D), Iranise Moro Pereira Jorge ${ }^{\mathrm{a}, \mathrm{d}}$ (D) \\ ${ }^{a}$ Universidade Federal do Paraná - UFPR, Curitiba, PR, Brasil. \\ ' Universidade Católica do Paraná - PUC-PR, PR, Brasil. \\ 'Associação dos Deficientes Físicos do Paraná - ADFP, Curitiba, PR, Brasil. \\ ${ }^{\mathrm{d}}$ Universidade Nova de Lisboa - Lisboa, Portugal.
}

Como citar: Sousa, A. C. S., Frasson, É. V. F., Kusma, S. Z., Vara, M. F. F., Fabri, A. F., \& Pereira Jorge, I. M. (2021). Identificação de papéis ocupacionais em atletas do esporte adaptado. Cadernos Brasileiros de Terapia Ocupacional, 29, e2131. https://doi.org/10.1590/2526-8910.ctoAO2131

\begin{abstract}
$\underline{\text { Resumo }}$
Objetivo: $\mathrm{O}$ presente estudo tem por objetivo descrever os papéis ocupacionais desempenhados pelos atletas da Associação dos Deficientes Físicos do Paraná ADFP. Método: Trata-se de uma pesquisa quantitativa observacional transversal. Foram realizadas entrevistas individuais estruturadas com base em uma Lista de Identificação de Papéis Ocupacionais - LIPO, na qual a amostra contou com os atletas vinculados a 6 modalidades oferecidas pela ADFP. Resultados: Participaram 37 atletas, com predominância do sexo masculino, cuja média de idade foi de 39 anos e o tempo de prática variou entre 1 mês e 25 anos. Destaca-se com significância os papéis ocupacionais de cuidador, membro da família e religioso sobre o desempenho do esporte. Conclusão: A pesquisa contou com boa adesão dos participantes, o que tornou possível verificar os papéis ocupacionais na vida dos atletas e a dinâmica de relação destes com seu desempenho no esporte. Da mesma forma, ressalta-se a necessidade de fomentar pesquisas relacionadas à temática, visto a incipiência de literatura nacional.
\end{abstract}

Palavras-chave: Atletas. Esportes para Pessoas com Deficiência. Desempenho de Papéis. Terapia Ocupacional. Análise e Desempenho de Tarefas.

\section{$\underline{\text { Abstract }}$}

Objective: The present study aims to define the occupational roles played by athletes from the Paraná Association of Physically Disabled - ADFP. Method: this is cross-sectional observational quantitative research. Individual interviews were carried out based on the Occupational Roles Identification List - ORIL, where the sample included athletes linked to the 6 modalities offered by ADFP. Results: The participants were 37 athletes, predominantly male, whose average age is 39 years old and the practice time varies between 1 month and 25 years. The occupational 
roles of caregiver, family member, and religious regarding the performance of the sport are highlighted with significance. Conclusion: The research had good participation by the participants, which enabled us to verify the occupational roles in the lives of the athletes and the dynamics of their relationship with their performance in the sport. Likewise, the need to encourage research related to the theme is emphasized, given the incipience of national literature.

Keywords: Athletes. Sports for Persons with Disabilities. Role Playing. Occupational Therapy. Task Performance, and Analysis.

\section{Introduçáo}

A prática profissional do terapeuta ocupacional é pautada no estudo da ocupação humana. O conceito surge em um dos pilares do Modelo de Ocupação Humana MOHO, descrito por Kielhofner (2002), na década de 70, e tem por finalidade resgatar e descrever o objeto de estudo da terapia ocupacional: a ocupação humana. Dentro disso, compreende-se que as necessidades de cada indivíduo são constituídas com base em diversos fatores intrínsecos e extrínsecos e que estes mesmos fatores, assim como influenciam no senso de capacidade, também estão ligados à sensação de frustração ou mesmo à tomada de decisão ao cessar alguma atividade (Kielhofner, 2002).

$\mathrm{O} \mathrm{MOHO}$ considera o humano como um sistema aberto, cuja organização interna depende de três subsistemas: a volição trata de valores, interesses e do conhecimento do sujeito relacionado à sua eficácia perante uma atividade; a habituação trata do hábito adquirido pela repetiçáo do comportamento, bem como do conjunto destes; enquanto a capacidade de desempenho faz o delineamento dos sistemas músculo-esquelético, neurológico e cardiopulmonar, além das imagens simbólicas, habilidades necessárias para a conquista do comportamento. Estes três conceitos garantem o desempenho satisfatório de papéis ocupacionais (Hagedorn, 2002).

Entende-se por papéis ocupacionais "conjuntos de comportamentos esperados pela sociedade e moldados pela cultura e contexto [...] que podem ser melhor conceituados pelo cliente" (Cavalcanti et al., 2015, p. 8). Segundo Parkinson et al. (2006), também estão incumbidas a estas expectativas, as quais dependem da competência do sujeito para cumpri-las de forma satisfatória de acordo com as circunstâncias vivenciadas.

Nesta perspectiva, o terapeuta ocupacional está apto a trabalhar atividades e, dentre elas, estimular o equilíbrio e o desempenho de papéis ocupacionais com diferentes populaçóes. Com base na reflexão geral da Resolução N495, de 18 de Dezembro de 2017, do Conselho Federal de Fisioterapia e Terapia Ocupacional - COFFITO, o desporto e paradesporto, campos ainda pouco explorados em cenário nacional, prevê como competências do profissional de terapia ocupacional avaliar, prescrever e orientar a prática do paradesporto (Conselho Federal de Fisioterapia e Terapia Ocupacional, 2018).

Neste contexto, encontra-se a Associação dos Deficientes Físicos do Paraná - ADFP, instituição de cunho filantrópico, sede de um Centro de Referência em Reabilitação Física com atendimentos de dança, enfermagem, fisioterapia, psicologia, serviço social e terapia ocupacional, além do setor de esporte adaptado. Este setor conta com 7 das 25 
modalidades reconhecidas pelo Comitê Paralímpico Internacional - CPI (Comitê Paralímpico Brasileiro, 2019), são elas: atletismo, basquete, bocha, esgrima, natação, tênis de mesa e tiro esportivo; contemplando o total de 59 atletas. Destes alguns se destacam por seu rendimento participando das paraolimpíadas. Percebe-se ainda o fomento em parcerias com algumas universidades, dando suporte ao processo de reabilitação e a prática do paradesporto.

Para posterior análise do impacto das intervençóes do terapeuta ocupacional sobre a prática do esporte adaptado, é necessária, inicialmente, a caracterização dos papéis ocupacionais desempenhados por estes atletas. Dado o contexto bibliográfico e o cenário da pesquisa, o presente estudo tem por objetivo descrever os papéis ocupacionais desempenhados pelos atletas da Associação dos Deficientes Físicos do Paraná.

\section{Metodologia}

Trata-se de uma pesquisa de caráter quantitativo, observacional, analítico transversal, sendo esta parte integrante de um projeto de pesquisa intitulado "Paradesporto, Reabilitação Física e Terapia Ocupacional”, que obteve aprovação pelo Comitê de Ética em Pesquisa em Seres Humanos sob o parecer no 2495358, em 15/02/2018, o qual garante o sigilo e anonimato da pesquisa para fins científicos e a assinatura do Termo de Consentimento Livre e Esclarecido - TCLE, conforme Resolução n ${ }^{\circ} 466 / 12$, do Conselho Nacional de Saúde - CNS.

Participaram da pesquisa 37 atletas de 6 modalidades: atletismo, basquete, esgrima, natação, tênis de mesa e tiro esportivo, os quais foram escolhidos por meio de amostragem por conveniência. Para a coleta de dados, utilizou-se dois instrumentos: um questionário sociodemográfico e a Lista de Identificação de Papéis Ocupacionais LIPO.

O primeiro questionário é semi-estruturado e foi desenvolvido pelas autoras, o qual busca descrever o indivíduo em relação aos dados pessoais, ocupacionais e interesses relacionados ao paradesporto. A LIPO é um instrumento validado e tem por finalidade obter a percepção do indivíduo acerca de sua participação nos principais papéis ocupacionais desenvolvidos ao longo de sua vida, bem como sua relevância. Esta está configurada em 10 papéis ocupacionais identificados por: estudante, trabalhador, voluntário, cuidador, serviço doméstico, amigo, membro da família, religioso, passatempo/amador, participante em organizaçôes e conta com a opção "outro", que possibilita ao indivíduo identificar um papel desempenhado não mencionado anteriormente (Cordeiro, 2005). A mesma autora orienta o preenchimento do instrumento, que é dividido em duas partes, sendo que a primeira avalia o desempenho ocupacional ao longo do presente (desde o contato com o esporte), passado (antes do contato como esporte) e futuro (o que espera para o futuro desde o momento da entrevista), permitindo que o participante assinale mais de uma variável por papel, enquanto a segunda parte determina a importância destes papéis - em nenhuma, alguma ou muita importância -, limitando a marcação de uma variável por papel.

As coletas foram realizadas no formato de entrevista individual nos locais de treino, de cada modalidade, durante o primeiro semestre de 2019, sendo necessário um encontro com média de 20 minutos por participante. As mesmas aconteceram 
conforme combinado com os treinadores e dependendo da disponibilidade de cada atleta, dando preferência para que acontecessem antes dos treinos, para evitar que o cansaço interferisse no resultado e que não prejudicasse a organizaçáo pessoal póstreino.

Para análise dos dados, foram contempladas as considerações e orientações da própria escala, bem como foi realizada uma exposição estatística dos dados por meio do Programa Computacional Statiscal Package for Social Sciences - SPSS, versão 22.0. As variáveis quantitativas foram analisadas por médias, medianas, valores mínimos, valores máximos, desvios padróes e a significância $(\mathrm{p}>0,05)$, segundo o teste qui-quadrado de Pearson.

\section{Resultados}

Ao se referir aos dados sociodemográficos (Tabela 1), participaram 37 atletas com os seguintes resultados: 8 mulheres e 29 homens, com idade entre 21 e 67 anos e média de 39 anos, com maior representatividade do tênis de mesa com 17 atletas. Dentre todos os atletas, $43,2 \%$ são casados, $51,4 \%$ possuem filhos, a média de 1 filho, e residem em Curitiba. Destes, 59,5\% apresentam diagnóstico de lesão medular e usam cadeira de rodas como meio exclusivo de locomoção. Observa-se que $86,48 \%$ dos participantes exerciam atividade remunerada antes de virem a praticar o esporte adaptado. No que diz respeito ao tempo de prática, que varia entre 1 mês e 25 anos, a maior frequência é a de 1 ano, que corresponde a $16,2 \%$.

Tabela 1. Dados sociodemográficos.

\begin{tabular}{ccccc}
\hline & Valor Mínimo & Valor Máximo & Média & Mediana \\
\hline Idade & 21 anos & 67 anos & 39,70 anos & 40 anos \\
\hline Filhos & 0 & 4 & 1 & 1 \\
\hline Tempo no Esporte & 0,1 ano & 25 anos & 7,41 anos & 6 anos \\
\hline
\end{tabular}

Fonte: As autoras.

Quanto à LIPO, os dados coletados estão apresentados em duas partes: 1- papéis ocupacionais desempenhados no passado, presente e futuro; 2- importância em sua realização.

Em relação à primeira parte (Tabela 2), pode-se notar o destaque do papel de estudante no passado como o único dos 11 papéis elencados que obteve pontuação máxima $(\mathrm{N}=37)$ e, em contrapartida, participante em organizaçóes foi o menos pontuado, com 7 indicaçóes. Quanto ao presente, os papéis mais pontuados são: serviço doméstico e membro da família; e os menos indicados foram os de estudante, voluntário e participante em organizaçóes. No que tange ao futuro, os papéis mais apontados foram os de membro da família, cuidador, serviço doméstico e passatempo, e o menos indicado é participante em organizaçôes. A opção outro foi considerada por 04 paratletas. 
Tabela 2. Papéis ocupacionais desempenhados no passado, presente e futuro.

\begin{tabular}{cccc}
\hline PAPEL & PASSADO & PRESENTE & FUTURO \\
\hline ESTUDANTE & 37 & 06 & 23 \\
\hline TRABALHADOR & 32 & 11 & 20 \\
\hline VOLUNTÁRIO & 12 & 09 & 27 \\
\hline CUIDADOR & 24 & 25 & 31 \\
\hline SERVIÇO DOMÉSTICO & 29 & 34 & 31 \\
\hline AMIGO & 29 & 28 & 30 \\
\hline MEMBRO DA FAMÍLIA & 32 & 32 & 35 \\
\hline RELIGIOSO & 30 & 18 & 28 \\
\hline PASSATEMPO/AMADOR & 28 & 26 & 31 \\
\hline PARTICIPANTE EM ORGANIZAÇÓES & 07 & 09 & 19 \\
\hline OUTRO & 02 & 02 & 04 \\
\hline
\end{tabular}

Fonte: As autoras.

Conforme a Tabela 2, é possível observar que os papéis de cuidador, serviço doméstico e passatempo/amador foram pontuados igualmente, ou seja, 31, por todos os participantes no futuro.

Quanto à segunda parte da LIPO (Tabela 3), os papéis destacados como "muita importância" foram os de membro da família, estudante, amigo e trabalhador, sendo o segundo e o terceiro igual a 26.

Observa-se que a maioria dos paratletas participantes da pesquisa apontam o score de “muita importância” aos papéis cuja execução exige/permite a presença de terceiros.

Tabela 3. Relação de importância dos papéis ocupacionais em pouca importância, alguma importância e muita importância.

\begin{tabular}{cccc}
\hline PAPEL & $\begin{array}{c}\text { POUCA } \\
\text { IMPORTÂNCIA }\end{array}$ & $\begin{array}{c}\text { ALGUMA } \\
\text { IMPORTÂNCIA }\end{array}$ & $\begin{array}{c}\text { MUITA } \\
\text { IMPORTÂNCIA }\end{array}$ \\
\hline ESTUDANTE & 03 & 08 & 26 \\
\hline TRABALHADOR & 05 & 08 & 24 \\
\hline VOLUNTÁRIO & 09 & 13 & 15 \\
\hline CUIDADOR & 06 & 08 & 23 \\
\hline SERVIÇO DOMÉSTICO & 05 & 09 & 23 \\
\hline AMIGO & 02 & 09 & 26 \\
\hline MEMBRO DA FAMÍLIA & 01 & 03 & 23 \\
\hline RELIGIOSO & 08 & 09 & 19 \\
\hline PASSATEMPO/AMADOR & - & 18 & 11 \\
\hline PARTIC. EM & 09 & 15 & 03 \\
\hline ORGANIZAÇÓES & - & - & 20 \\
\hline OUTRO & & & \\
\hline
\end{tabular}

Fonte: As autoras.

Observa-se maior discrepância nas respostas ao papel de serviço doméstico em relação ao desempenho no passado, presente e futuro (Tabela 2) ao ser comparado com seu grau de importância na Tabela 3. 
Tabela 4. Cruzamento das variáveis de papel desempenhado no presente x muita importância.

\begin{tabular}{|c|c|c|c|c|c|}
\hline \multirow[t]{2}{*}{ PAPEL } & \multicolumn{2}{|c|}{ PRESENTE } & \multicolumn{2}{|c|}{$\begin{array}{c}\text { MUITA } \\
\text { IMPORTÂNCIA }\end{array}$} & \multirow{2}{*}{$\begin{array}{c}\text { VALOR DE P } \\
(>0,05)\end{array}$} \\
\hline & $\mathbf{n}$ & $\%$ & $\mathbf{n}$ & $\%$ & \\
\hline ESTUDANTE & 06 & 16,21 & 5 & 83,3 & 0,30 \\
\hline TRABALHADOR & 11 & 29,72 & 10 & 90,9 & 0,08 \\
\hline VOLUNTÁRIO & 09 & 24,32 & 5 & 55,6 & 0,46 \\
\hline CUIDADOR & 25 & 67,56 & 20 & 80 & 0,00 \\
\hline SERVIÇO DOMÉSTICO & 34 & 91,89 & 23 & 67,6 & 0,06 \\
\hline AMIGO & 28 & 75,67 & 21 & 75 & 0,47 \\
\hline MEMBRO DA FAMÍLIA & 32 & 86,48 & 30 & 93,8 & 0,01 \\
\hline RELIGIOSO & 18 & 48,64 & 16 & 88,9 & 0,00 \\
\hline PASSATEMPO/AMADOR & 26 & 70,27 & 16 & 61,5 & 0,05 \\
\hline $\begin{array}{c}\text { PARTIC. EM } \\
\text { ORGANIZAÇÔES }\end{array}$ & 09 & 24,32 & 3 & 33,3 & 0,47 \\
\hline OUTRO & 02 & 5,40 & 2 & 100 & - \\
\hline
\end{tabular}

Fonte: As autoras.

No que diz respeito à análise de dados pelo programa SPSS, é possível identificar que os papéis com maior relevância $(\mathrm{p}<0,05)$ são os de religioso $(\mathrm{p}<0,01)$, cuidador ( $\mathrm{p}<0,01)$ e membro da família $(\mathrm{p}=0,01)$ e, em contrapartida, com menor relevância tem-se os papéis de participante em organizaçóes $(p>0,05)$, amigo $(p>0,05)$ e voluntário $(\mathrm{p}>0,05)$.

\section{Discussão}

Em relação aos dados sociodemográficos, o presente estudo conta com uma amostra predominantemente masculina, sendo 29 homens para 8 mulheres. Camargo \& Kessler (2017) consideram o esporte como um mundo masculino que apresenta barreiras de gênero que dificultam o ingresso e permanência de corpos femininos ou, ainda, de homossexuais ou em transição de gênero. Corroborando o achado, o Instituto Noos de Pesquisas Sistêmicas e Desenvolvimento de Redes Sociais (2007) traz que por muito tempo as mulheres foram proibidas de praticarem esportes por estes serem considerados incompatíveis e inadequados para o gênero. Embora muitos paradigmas tenham sido rompidos, resquícios ainda são encontrados, fazendo com que este seja ainda um aspecto a ser discutido e explorado.

Em relação ao diagnóstico, observa-se que mais da metade da amostra, 59\%, possui Lesão Medular - LM, condição que acaba por submeter o indivíduo a diversos deficit que podem variar desde os estruturais aos funcionais por uma série de circunstâncias, sejam elas oriundas da própria patologia ou por lesóes secundárias, como por inadequação de posicionamento, por exemplo. Além destes, uma lesão grave pode, ainda, dificultar ou até mesmo cessar o desempenho de papéis ocupacionais, exigindo que o indivíduo passe por um processo de readequação e aquisição de novas formas de ser e fazer (Souza et al., 2013; Luzo et al., 2004; Kielhofner, 2009). 
É notório que existem diversos benefícios com a prática esportiva às pessoas com LM, podendo ser destacados inicialmente o ganho de capacidade aeróbica e do consumo máximo de oxigênio, a redução do risco de doenças cardiovasculares e de infecçóes respiratórias, e diminuição na incidência de complicaçôes médicas. Estes ganhos potencializam os níveis de integração comunitária e participação, intensificam a independência e a autoimagem, auxiliando no enfrentamento da deficiência, favorecendo assim o aumento da satisfação e expectativa de vida (Silva et al., 2005; Medola et al., 2011).

É relevante apontar que a reflexão desenvolvida teve pano de fundo no esporte, pois a amostra está intimamente ligada a esse cenário, possibilitando um diálogo do seu cotidiano. Sendo assim, a primeira parte da LIPO foi direcionada com base neste: passado como sendo antes do contato com o esporte; presente como sendo desde o primeiro contato; e futuro como sendo desde o momento da entrevista. Porém, como este estudo apresentou delineamento transversal, não foi possível estabelecer uma relação de causa e efeito entre os papéis ocupacionais e o esporte.

Com base na análise do cruzamento dos dados das partes I e II da LIPO (Tabela 4), os papéis de cuidador, membro da família e religioso apresentam significância por meio do valor de $\mathrm{p}(\mathrm{p}<0,05)$.

Na perspectiva dos papéis apontados com maior ênfase na pesquisa, Aizava (2011) defende que estes possibilitam o suporte social por terem como principal característica o envolvimento em atividades realizadas com determinada frequência e que compreendem a coparticipação de pares que podem ou não apresentar vínculo familiar.

O mesmo autor descreve este suporte como sendo constituído por redes de comunicação em que há emprego de valores mútuos e como influenciador da performance do sujeito no esporte adaptado sob variação de $24 \%$, além dos impactos causados à diminuição do estresse, às manifestaçôes comportamentais, cognitivas e afetivas (Aizava, 2011). O achado corrobora o estudo de Cruz (2012), que destaca a família como potencializadora deste suporte.

A volição, primeiro conceito formador do $\mathrm{MOHO}$, está diretamente ligada ao significado que o sujeito atribui à atividade em questão e sofre forte influência dos contextos cultural e familiar (Kielhofner, 2002). Em um estudo realizado com 114 jovens em idade escolar praticantes de diversas modalidades de esporte adaptado, a família é posta como um dos principais fatores para o ingresso e permanência do sujeito enquanto atleta, o que tem se potencializado diante do cenário mundial atual, no qual os esportes paralímpicos têm ganhado cada vez mais espaço diante da mídia. Ressaltase, ainda, que tal incentivo está diretamente ligado à autoimagem e à motivação do sujeito devido à sensação de ser orgulho no seio familiar (Resende et al., 2019).

Segundo Mizoguchi et al. (2013) e Aizava (2011), as relaçóes familiares estão diretamente ao que chamam de suporte emocional: aquele que vem como potencializador do desempenho na vida cotidiana e estimulam a autoconfiança do suporte. Neste estudo, apresentam ainda correlação positiva deste com o desempenho dos sujeitos na prática desportiva, bem como o contrário (Aizava, 2011; Mizoguchi et al., 2013).

Segundo Haiachi et al. (2016), há que se considerar ainda outros fatores interligados a esta dinâmica. Para os autores, o ambiente esportivo apresenta três tipos de barreiras: condiçôes ambientais (arquitetônicas), estruturais (financeiras e inerentes à modalidade) 
e emocionais, as quais estão associadas às relações familiares e também àquelas que são construídas neste contexto. No que diz respeito à prática de rendimento, cenário no qual o presente estudo se constituiu, estes contrapóem as duas últimas barreiras como sendo também facilitadores à medida em que o atleta se destaca na modalidade.

A religiosidade, outro papel no qual foram encontrados resultados expressivos, está interligada à proximidade entre os valores e princípios incumbidos ao papel com o esporte (Ferreira, 2010). Observou-se, por meio do valor de $\mathrm{p}(\mathrm{p}=<0,01)$, que a extrema significância pode estar relacionada também ao que o autor disserta sobre a atribuição do significado de santuário ao corpo, que passa a ser um sujeito comum de cuidado entre esporte e religião (Ferreira, 2010). Subjetivamente, este papel está incluso também nas ideias apresentadas por Aizava (2011) e Mizoguchi et al. (2013) quando a análise parte do ponto de vista do suporte oferecido por grupos sociais criados nos ambientes de prática religiosa, os quais fortalecem e são fortalecidos pela prática desportiva.

Considera-se que a terapia ocupacional pode contribuir no campo do Desporto e Paradesporto para além do treino de habilidades, prescrição, confecção e treinamento de tecnologias assistivas, fazendo-se área profissional fundamental para a constituição da equipe multidisciplinar como catalisador do desempenho ocupacional e, ainda, para o fortalecimento destas redes de apoio.

\section{Consideraçóes Finais}

O esporte paralímpico começa a ganhar espaço como objeto de estudo no Brasil na década de 90. Em 2001, segundo Gorla \& Nogueira (2016), os profissionais da educação física passaram a se dedicar continuamente à profissionalização nas modalidades no país. Mesmo que terapeutas ocupacionais viessem desenvolvendo açóes junto ao esporte adaptado anteriormente, apenas em 2017 o COFFITO legitimou o terapeuta ocupacional como profissional atuante no campo. Todavia, em virtude desta regulamentação ainda recente, as produções científicas são ainda incipientes, fazendo com que os profissionais acabem por embasar suas práticas por ciências afins.

No contexto da pessoa com deficiência física ou mobilidade reduzida, neste caso os atletas do esporte adaptado, muitos acabam enfrentando barreiras arquitetônicas e atitudinais, deficit relacionados ao desempenho ocupacional e à influência direta em sua idiossincrasia, bem como seu modo de interagir com o mundo, na relação com o próprio corpo e com o outro, identificando aí várias possibilidades de atuação para a terapia ocupacional.

Considerando a dimensão que este alcança e acreditando na participação ocupacional e social dessas pessoas, o serviço de terapia ocupacional, neste contexto, busca promover a integração em vários momentos, a fim de utilizar o desporto como facilitador do desempenho ocupacional, baseando-se no princípio de que sua prática potencializa o desenvolvimento de habilidades, o processo terapêutico e o desempenho de papéis ocupacionais. Para tanto, é necessário mapear/identificar os papéis ocupacionais atuais e desejados (presente e futuro), para depois desenvolver e correlacionar as açóes da terapia ocupacional com o esporte.

Quanto ao desenvolvimento da pesquisa, considera-se que o objetivo foi alcançado. Além disso, ressalta-se a preocupaçáo no que diz respeito à descrição de papéis ocupacionais pelo avaliador, no momento da entrevista, dado o fato de que a prática do 
esporte, neste caso, foi interpretada como o papel de trabalhador, passatempo/amador e, ainda, preencher o espaço "outro". O mesmo pode acontecer com outros papéis desempenhados pelo participante, comprometendo a análise dos resultados.

\section{REFERÊNCIAS}

Aizava, P. V. S. (2011). Autoeficácia, qualidade de vida e suporte social relacionados ao esporte de rendimento (Dissertação de mestrado). Universidade Estadual de Maringá, Maringá.

Camargo, W. X., \& Kessler, C. S. (2017). Além do Masculino/Feminino: gênero, sexualidade, tecnologia e performance no esporte sob perspectiva crítica. Horizontes Antropológicos, 47, 191-225.

Cavalcanti, A., Dutra, F. S., \& Elui, V. (2015). Estrutura da prática de Terapia Ocupacional: domínio e processo. Revista de Terapia Ocupacional da Universidade de São Paulo, 26, 1-49. http://dx.doi.org/10.11606/issn.2238-6149.v26iespp1-49.

Conselho Federal de Fisioterapia e Terapia Ocupacional - COFFITO. (2018). Resolução n495, de 18 de dezembro de 2017. Disciplina a atuação profissional da Terapia Ocupacional no desporto e paradesporto e dá outras providências. Diário Oficial [da] República Federativa do Brasil, Brasília, seção 1, p.141-142.

Comitê Paralímpico Brasileiro (2019). História. Recuperado em 26 de junho de 2019, de https://www.cpb.org.br.

Cordeiro, J. J. R. (2005). Validação da Lista de Identificação de Papéis Ocupacionais em pacientes portadores de doença pulmonar obstrutiva crônica (DPOC) no Brasil (Dissertação de mestrado). Universidade Federal de São Paulo, São Paulo.

Cruz, D. M. C. (2012). Papéis Ocupacional e Pessoas com Deficiências Físicas: independência, tecnologia assistiva e poder aquisitivo (Tese de doutorado). Universidade Federal de São Carlos, São Carlos.

Ferreira, L. V. O. (2010). Educação Física, esporte e religiāo: interferências e relaçôes. (Monografia de licenciatura). Universidade Federal de Minas Gerais, Belo Horizonte.

Gorla, J. I., \& Nogueira, C. D. (2016). Esporte Adaptado e Formação de Recursos Humanos: esperiências contruídas na área. Conexões, 14(2), 158-164.

Hagedorn, R. (2002). Modelos de Terapia Ocupacional. In R. Hagedorn, Fundamentos da Prática em Terapia Ocupacional (pp.151-159). São Paulo: Dynamis Editorial.

Haiachi, M. C., Cardoso, V. D., Reppold Filho, A. R., \& Gaya, A. C. A. (2016). Reflexóes sobre a carreira do atleta paraolímpico brasileiro. Ciência \& Saúde Coletiva, 21(10), 2999-3006. http://dx.doi.org/10.1590/1413-812320152110.18512016.

Instituto Noos de Pesquisas Sistêmicas e Desenvolvimento de Redes Sociais - INPSDRS. (2007). A Mulher e o Esporte: a experiência dos municipios do Rio de Janeiro e São Paulo. Recuperado em 26 de junho de 2019, de https://noos.org.br/docs/resumofinal_port.pdf.

Kielhofner, G. (2009). Conceptual Foundations of Occupational Therapy. Philadelphia: FA Davies Company.

Kielhofner, G. (2002). Model of Fuman Occupationa: theory and application. Baltimore: Williams \& Wilkins.

Luzo, M. C. M., Lourenção, M. I. P., \& Elui, V. M. C. (2004). Atuação Terapêutico-ocupacional Junto a Pacientes com Comprometimentos Traumato-ortopédicos. In M. M. R. P. De Carlo \& M. C. M. Luzo (Orgs.), Terapia Ocupacional: reabilitação física e contextos hospitalares (pp. 145-152). São Paulo: Roca.

Medola, F. O., Busto, R. M., Marçal, A. F., Achour Junior, A., \& Dourado, A. C. (2011). O esporte na qualidade de vida de indivíduos com lesão da medula espinhal: série de casos. Revista Brasileira de Medicina do Esporte, 17(4), 254-256. http://dx.doi.org/10.1590/S1517-86922011000400008. 
Mizoguchi, M. V., Balbim, G. M., \& Vieira, L. F. (2013). Estilo parental, motivação e satisfação de atletas de beisebol: um estudo correlacional. Revista da Educação Física/UEM, 24(2), 215-223. http://dx.doi.org/10.4025/reveducfis.v24.2.16282.

Parkinson, S., Forsyth, K., \& Kielhofner, G. (2006). User's manual for the Model of Human Occupation Screening Tool (MOHOST) version 2.0. Chicago: University of Illinois.

Resende, M. C., de Carvalho-Freitas, M. N., \& Guimarães, A. C. (2019). Percepçôes sobre as paralimpíadas escolares: um estudo com atletas. Pensar a Prática, 22, 1-12. http://dx.doi.org/10.5216/rpp.v22.51476.

Silva, M. C. R., Oliveira, R. J., \& Conceição, M. I. G. (2005). Efeitos da Natação sobre a Independência Funcional de Pacientes com Lesão Medular. Revista Brasileira de Medicina do Esporte, 11(4), 251256.

Souza, F. D. A., Cruz, D. M. C., Ferrigno, I. S. V., Tsukimoto, G. R., \& Figliolia, C. S. (2013). Correlação entre papéis ocupacionais e independência de usuários com lesão medular em processo de reabilitação. O Mundo da Saúde, 37(2), 166-175.

\section{Contribuição dos Autores}

Ana Cláudia da Silva de Sousa: Concepção do texto, organização das fontes e redação. Érika Vanessa Freire Frasson: Concepção do texto e redação. Solena Ziemer Kusma: Organização de análises. Maria de Fátima Fernandes Vara: Concepçáo do texto e revisão do texto. Aline Ferrari Fabri: Concepçáo do texto e revisão do texto. Iranise Moro Pereira Jorge: Concepção do texto, organização de análise, redação e revisão do texto. Todas as autoras aprovaram a versão final do artigo.

\section{Autor para Correspondência}

Iranise Moro Pereira Jorge

E-mail: ira.mpj@gmail.com

\section{Editora de Seção}

Profa. Dra. Daniela Tavares Gontijo 